\title{
Análise de agentes patogênicos em fezes de pombos da Cidade de Campina Grande -
}

\section{PB}

\author{
Analysis of pathogens in feces of palomas in the City of Campina Grande - PB \\ Análisis de agentes patógenos en heces de palomas de la Ciudad de Campina Grande - PB
}

Recebido: 26/07/2021 | Revisado: 20/07/2021 | Aceito: 10/08/2021 | Publicado: 13/08/2021

\author{
Kênia Kelly Freitas Sarmento \\ ORCID: https://orcid.org/0000-0001-6815-9577 \\ Universidade Estadual da Paraíba, Brasil \\ E-mail: keniakellys41@gmail.com \\ José Everton Soares de Souza \\ ORCID: https://orcid.org/0000-0003-0731-534X \\ Universidade Estadual da Paraíba, Brasil \\ E-mail: everton_g3@hotmail.com \\ Vanessa Rosales Bezerra \\ ORCID: https://orcid.org/0000-0002-7920-4107 \\ Universidade Estadual da Paraíba, Brasil \\ E-mail: rosalesuepb@gmail.com \\ Karyna Steffane da Silva \\ ORCID: https://orcid.org/0000-0002-2048-372X \\ Universidade Estadual da Paraíba, Brasil \\ E-mail: karynasteffane@ hotmail.com \\ Keila Machado de Medeiros \\ ORCID: https://orcid.org/0000-0001-9250-1432 \\ Universidade Federal do Recôncavo da Bahia, Brasil \\ E-mail: keilamedeiros@ufrb.edu.br \\ Carlos Antônio Pereira de Lima \\ ORCID: https://orcid.org/0000-0002-1301-6066 \\ Universidade Estadual da Paraíba, Brasil \\ E-mail: caplima@uepb.edu.br
}

\begin{abstract}
Resumo
Pombos que partilham o mesmo espaço dos humanos aparentam serem seres inofensivos a saúde, todavia, estas aves representam um agravo para saúde pública, tendo em vista que possuem a capacidade de disseminar doenças através de suas fezes, sendo uma delas a criptococose, da espécie Columba livia. Por motivos de ter contato com as pessoas, para se alimentarem pode aumentar a disseminação de agentes patogênicos para os humanos. Diante desta pesquisa, o presente estudo tem como objetivo fazer um levantamento bibliográfico sobre algumas doenças propagadas por pombos e uma analise da presença de patógenos em fezes de pombos urbanos presentes na cidade de Campina Grande - PB, através de investigação laboratorial, com finalidade de sugestões e de despertar à população a respeito de riscos á saúde e prejuízos, objetivando a adoção de padrões eficazes de controle. Foram coletadas quatro amostras de excretas de pombos e enviadas para análise laboratorial. A coleta foi conduzida em dois pontos que apresentam grande infestação de pombos, toda a coleta foi realizada com o uso de materiais descartáveis e higienizados, por conseguinte devidamente refrigerados para manter a integridade das amostras. Não foram encontradas presença de agentes patogênicos na amostra investigada, porém, esta quantidade de amostra foi bastante limitada, sendo necessários estudos futuros com um maior número de excretas deste tipo de ave.
\end{abstract}

Palavras-chave: Pombos; Fezes de pombos; Agentes patogênicos; Criptococose; Saúde pública.

\begin{abstract}
Pigeons that share the same space as humans appear to be harmless to health, however, these birds represent a problem for public health, given that they have the ability to spread diseases through their feces, one of which is cryptococcosis, of the species Columba livia. For reasons of having contact with people, to feed themselves can increase the spread of pathogenic agents to humans. In view of this research, this study aims to make a bibliographical survey on some diseases propagated by pigeons and an analysis of the presence of pathogens in the feces of urban pigeons present in the city of Campina Grande - PB, through laboratory investigation, with the purpose of making suggestions and to alert the population about health risks and losses, aiming at the adoption of effective control standards. Four samples of pigeon excreta were collected and sent for laboratory analysis. The collection was carried out in two points that present great infestation of pigeons, all the collection was carried out with the use of disposable and sanitized materials, therefore properly refrigerated to maintain the integrity of the samples. We did not find the presence of pathogenic agents in the
\end{abstract}


investigated sample, however, this amount of sample was quite limited, and future studies are needed with a greater number of excreta of this type of bird.

Keywords: Pigeons; Pigeon droppings; Pathogenic agents; Cryptococcosis; Public health.

\begin{abstract}
Resumen
Las palomas que comparten el mismo espacio que los humanos parecen ser inofensivas para la salud, sin embargo, estas aves representan un problema para la salud pública, dado que tienen la capacidad de transmitir enfermedades a través de sus heces, una de las cuales es la criptococosis, de la especie Columba livia. . Por motivos de tener contacto con personas, alimentarse por sí mismos puede incrementar la propagación de agentes patógenos a los humanos. En vista de esta investigación, este estudio tiene como objetivo realizar un relevamiento bibliográfico sobre algunas enfermedades propagadas por palomas y un análisis de la presencia de patógenos en las heces de palomas urbanas presentes en la ciudad de Campina Grande - PB, mediante investigación de laboratorio, con el propósito de hacer sugerencias y alertar a la población sobre riesgos y pérdidas para la salud, con el objetivo de adoptar estándares de control efectivos. Se recolectaron cuatro muestras de excrementos de palomas y se enviaron para análisis de laboratorio. La recolección se realizó en dos puntos que presentan gran infestación de palomas, toda la recolección se realizó con el uso de materiales desechables y desinfectados, por lo tanto debidamente refrigerados para mantener la integridad de las muestras. No encontramos la presencia de agentes patógenos en la muestra investigada, sin embargo, esta cantidad de muestra fue bastante limitada, y se necesitan estudios futuros con una mayor cantidad de excretas de este tipo de ave.

Palabras clave: Palomas; Excrementos de paloma; Agentes patógenos; Criptococosis; Salud pública.
\end{abstract}

\title{
1. Introdução
}

Os pombos considerados urbanos pertencem à ordem dos Columbiformes, foram introduzidos no Brasil por volta do século XVI, sendo consideradas aves domesticas, quando existe uma enorme disponibilidade de alimentos e locais para se abrigar, eles conseguem se adaptar facilmente nas cidades, podendo ser considerados como aves de estimação. Com a grande concentração destes pombos, considera-se um risco à saúde pública. (Kahn et al., 2012). Possuindo aproximadamente $35 \mathrm{~cm}$ de comprimento, com envergadura cerca de $60 \mathrm{~cm}$, com peso aproximado de $400 \mathrm{~g}$. Podendo ter cor branca e preta, existindo fartura de alimentos é considerado um fator primordial para os bandos se abrigarem e se reproduzirem rapidamente. Dentro de um modo geral os que compartilham o mesmo espaço do ambiente humano são considerados pertencentes à espécie Columba livia (Labanhare \& Perrelly, 2007; Martins, 2012).

A espécie Columba livia chegou ao Brasil na época da colonização portuguesa, e hoje comumente encontra-se a presença deste tipo de ave nos centros urbanos brasileiros, sendo um problema ambiental e de saúde pública em algumas cidades brasileiras (Araújo et al., 2010). Esse fenômeno pode ser explicado pelo fato de que a estrutura urbana oferece condições necessárias para a sua adaptação e reprodução, visto que, o ambiente fornece a alimentação por meio do lixo e/ou restos de alimentos acumulados, ou através de alimentos fornecidos pelo homem (Nunes \& Miranda, 2010). Ambientes ricos em fontes de nitrogênio favorecem o crescimento destes fungos (Pizani \& Santos, 2017).

Em conformidade com os autores Amâncio et al., (2008) cita que a Columba livia foi registrada com maior quantidade em diversas áreas de maior intensidade de setor urbano destacando intensidade de pessoas, praças, prédios e grande movimentação de veículos.

Segundo Schuller (2005) para ocasionar a infestação de pombos, o alimento é considerado um fator limitante para ocorrer desequilíbrio ambiental, onde o bando tende a ocupar. E se tem maior disponibilidade de alimento maior será a capacidade reprodutiva da espécie, podendo variar de 10 a 14 ovos por fêmea a cada ano, sendo em torno de 4 a 6 anos. Beck (2003) relatou que existindo condições para sobrevivência e alimentação podem ocorrer até seis ninhadas anualmente. De acordo com Torres et al., (2015) devido a ausência de um programa que seja voltado para o controle dessa espécie, o tempo de incubação é por volta de 17 a 19 dias, cada casal de pombos põe até dois ovos por ninhada.

Existe uma relação entre a figura do pombo com o símbolo de paz, religião e amor, assim, o animal não é tido como uma ameaça. Todavia, no século passado, Weber já tinha descrito 57 patologias ligadas a esta ave, que vão desde aquelas que afetam apenas aves até aquelas que atingem o ser humano (Contin et al., 2010). 
Diante deste cenário, tendo alimento e locais para os pombos se abrigarem, tem por finalidade reprodução da espécie de pombos de forma acelerada, no ponto de vista de estudo epidemiológico, eles podem conter vários tipos de microrganismos que são considerados danosos para o ser humano (Moutinho et al., 2013; Carrasco et al., 2011). Nesta condição esta ave é considerada como prejudicial à saúde pública, podendo contaminar o meio ambiente e transmitir doenças através das suas fezes ressecadas em suspensão que por sua vez podem ser inaladas, pelo contato coma as pessoas, devido a procura por alimentação, aumentando possibilidades de transmissão de agentes patogênicos para os humanos (Moutinho et al., 2015; Silva \& Capuano, 2008; Farias, 2016).

Oliveira \& Machado (2009) apontam que as doenças transmissíveis podem ser adquiridas por espécies de pombos exóticos que podem ser propagadas por organismos que por sua vez são originários de populações que possuem resistência a elas. Desta forma os pesquisadores relatam a falta de soluções e medidas para combater as proliferações geradas pelos pombos.

De acordo com uma inspeção realizada pelos autores Miranda et al., (2014), diante de entrevistas realizadas perceberam que a maior parte das pessoas reconhecem os pombos como disseminadores zoonoses, cerca de 73,4\% não conhecem o nível de transmissão de agentes patogênicos ao homem.

Destaca-se a via respiratória como meio de infecção através das excretas dos pombos, aspirando a poeira das fezes secas ou pela ingestão de alimentos que estão contaminados. Considerando um agravo a saúde publica as doenças obtidas com o contato direto com esses pombos, algumas delas são: Criptococose, Toxoplasmose, Psitacose e Salmonelose, de acordo com os estudos de (Mezzari et al., 2014; Silva \& Capuano, 2008).

De acordo com os autores observaram que houve um aumento em relação a infecções fúngicas gravemente relacionadas por fungos oportunistas, relatando crescimento da população geriátrica até mesmo infecções pelo HIV. (Aguiar et al., 2017; Silva et al., 2021; Araújo et al.,2021).

A criptococose, obtida por meio da inalação de aerossóis de excretas secas contaminadas, representa um fator de mortalidade, com casos já registrados no Estado do Paraná (Martins et al., 2016). A doença é transmitida através de um fungo leveduriforme encapsulado que recebe o nome de Cryptococcus neoformans. A levedura pode ser isolada em fezes de vários animais. No cenário contemporâneo, a enfermidade possui determinada relevância sendo tida como uma das micoses mais comuns em indivíduos imunodeprimidos (Cruz et al., 2015). A influência do Cryptococcus neoformans dentro da doença animal deve-se ao fato da interação em processos cutâneos, respiratórios, nervosos, ósseos e mamários. Ainda que o pulmão seja o primeiro a ser acometido pela criptococose, a mesma é uma doença do sistema nervoso central, visto que, geralmente o fungo atinge as meninges durante a disseminação (Fraga, 2014; Barbosa Junior et al., 2013).

Nas aves podem ser achados determinadas bactérias e fungos. Dentre eles destaca-se o gênero Cryptococcus que causa a criptococose, caracterizada por provocar agravo pulmonar principalmente em indivíduos com a imunidade baixa, sua disseminação se dar através das excretas dos pombos além dos fungos Aspergillus fumigatus e Histoplasma capsulatum, que causam problema respiratório principalmente em pacientes com Imunodeficiência Humana Adquirida (SIDA) (Neto et al., 2020; Aguiar et al., 2011; Ribeiro et al., 2017; Marques et al., 2010).

Diante desta pesquisa, o presente estudo tem como objetivo fazer um levantamento bibliográfico sobre algumas doenças propagadas por pombos e uma analise da presença de patógenos em fezes de pombos urbanos presentes na cidade de Campina Grande - PB, através de investigação laboratorial, com finalidade de sugestões e de despertar à população a respeito de riscos á saúde e prejuízos, objetivando a adoção de padrões eficazes de controle.

\section{Metodologia}

Toda coleta de dados foi realizada na Campina Grande - PB, no dia 23 de novembro de 2020, por volta das 09h30min da manhã, tendências anuais e sazonais de temperatura do ar, neste período de novembro encontra-se com presença de sol e 
pouca umidade. Assim, foram listados os locais da cidade com maior concentração de pombos, a Praça da bandeira em duas áreas diferentes, Rua Venâncio Neiva, e Rua Cardoso Vieira, contudo, foram escolhidos os locais que apresentam maior trânsito de pessoas e consequentemente pontos que apresentam maior propensão para acometimento de alguma doença proveniente do contato com as aves, assim, para delineamento do estudo, selecionou-se a Praça da Bandeira e na Rua Cardoso Vieira, dois lugares de acentuada movimentação de habitantes, pois estão situados na cidade de Campina Grande - PB, localizada no centro comercial.

O desenvolvimento da problemática do presente estudo foi sustentado através da literatura científica, assim, primordialmente foram analisadas as produções científicas no formato de artigos científicos e monografias, anexadas nas bases de dados Google Acadêmico e Scielo, sendo utilizadas como critério de inclusão, produções científicas que estão nos temas citados nos últimos anos, buscando um principio teórico, compreendendo o envolvimento das doenças disseminadas para os seres humanos através das excetas dos pombos.

Após a formulação das hipóteses e formação de uma problemática, foram iniciadas as coletas. Inicialmente se coletou quatro amostras, todavia, apenas duas foram armazenadas e encaminhadas para análise laboratorial, à limitação no número de amostra ocorreu devido aos custos inerentes à pesquisa, levando em consideração que a pesquisa contou apenas com financiamento próprio, não tendo patrocínio de nenhuma entidade pública ou privada. Dessa forma, a pesquisa possui um enquadramento eminentemente de abordagem qualitativa.

Nosso aporte metodológico é composto por vários autores dentre eles, (Contin et al., 2011; Neto et al., 2020; Silva \& Capuano, 2008; Farias, 2016; Labanhare \& Perrelly, 2007; Nunes \& Miranda, 2010; Nunes, 2003). Entretanto, teve sua essência baseada nas premissas propostas por Mezzari et al., (2014).

As fezes foram coletadas em pontos de contato dos pombos sendo escolhidas janelas de propriedades localizadas nos dois pontos selecionados, visto que existe uma maior probabilidade de contato humano nesses lugares. A coleta das fezes das aves se utilizou espátulas de madeira descartáveis e devidamente esterilizadas e armazenadas em tubos de plásticos, por conseguinte os tubos foram rotulados e devidamente armazenados, ou seja, foram refrigerados em um recipiente adequado, no intuito de preservar o material de modo que não exista um comprometimento dos resultados. A análise laboratorial foi realizada pelo laboratório Hermes Pardini, situado no município de Vespasiano - MG, já que nenhum laboratório do estado da Paraíba realizava essa análise.

\section{Resultados e Discussão}

Em conformidade com o delineamento de estudos foram escolhidos dois locais do centro urbano de Campina Grande PB, a Praça da Bandeira e a Rua Cardoso Vieira, que são pontos visíveis de infestação dos pombos, conforme as Figuras 1 e 2. 
Research, Society and Development, v. 10, n. 10, e387101018717, 2021

(CC BY 4.0) | ISSN 2525-3409 | DOI: http://dx.doi.org/10.33448/rsd-v10i10.18717

Figura 1. Praça da Bandeira.


Fonte: Autores (2020).

Figura 2. Rua Cardoso Vieira.
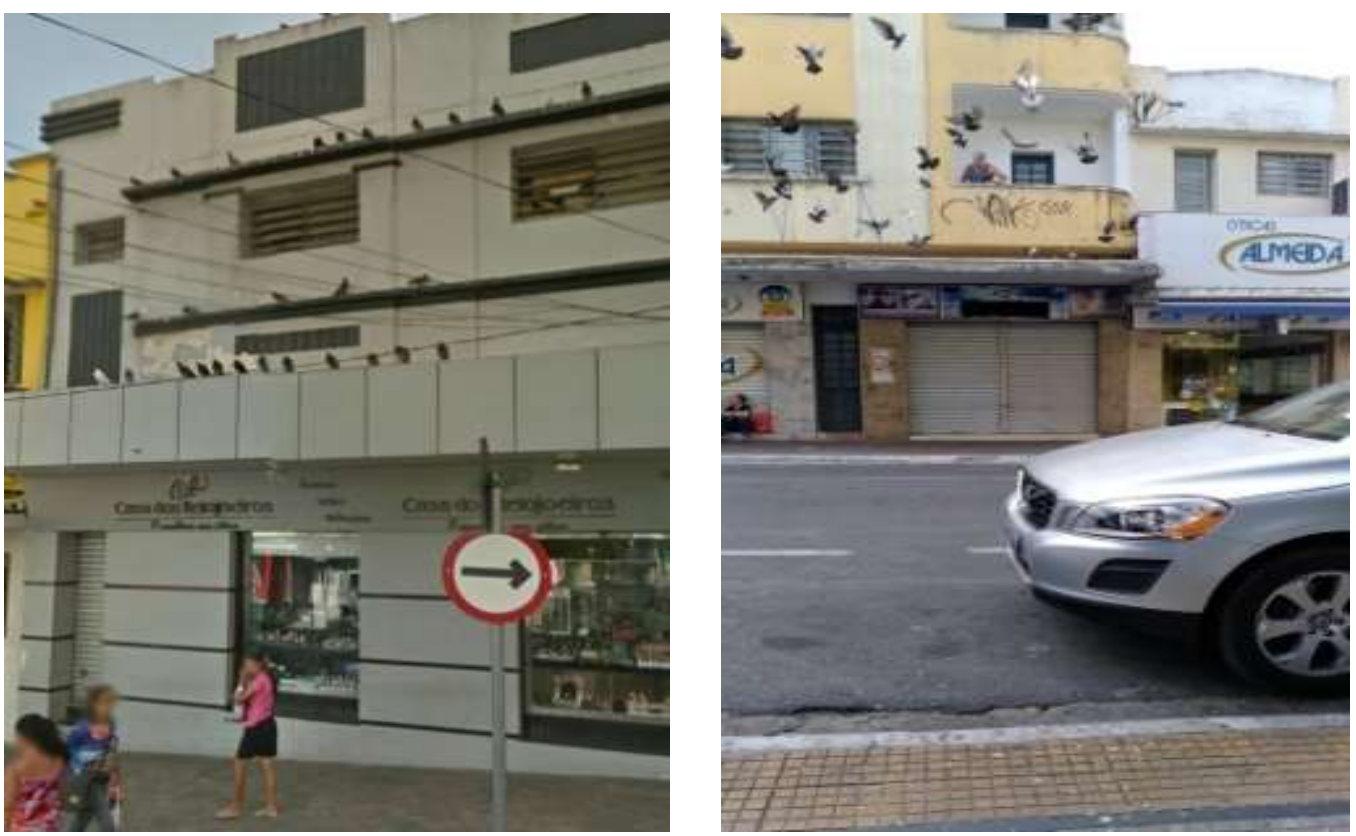

Fonte: Autores (2020).

Devido ao acúmulo de excretas dos pombos juntamente com penas levam a grandes prejuízos no sistema de drenagem quando a água escorre fazendo com que escorra e acontecendo o entupimento do sistema de drenagem, além dos microrganismos patogênicos disseminados por estes pombos, através principalmente das excretas.

A criptococose em pessoas com SIDA geralmente apresenta quadro clínico inespecífico, o que dificulta a orientação do médico para diagnosticar precocemente a doença, porém alguns sinais e sintomas são predominantemente relatados na literatura. 
Indivíduos com criptococose pulmonar relatam queixas respiratórias como tosse e dispneia, além de achados como infiltrado difuso e nódulos, que são confundidos com tuberculose. (Pizani \& Santos, 2017; Takahara, 2007; Silva \& Gagliani, 2014). A infecção pulmonar por Cryptococcus spp. se não diagnosticada a tempo pode levar à morte por insuficiência pulmonar (Leite et al., 2012).

Para Albano (2009), pesquisas voltadas a investigação das doenças infecciosas provenientes de animais silvestres, com ênfase para aquelas transmissoras de fungos, tem um baixo número de relatos quando associados a sua incidência e a segmentação de distintos agentes etiológicos nas populações de vida livre.

As espécies de pombos que habitam o mesmo espaço dos humanos aparentemente são animais inofensivos, porém, tem a capacidade de transmitir 30 doenças para o ser humano. Estas aves disseminam enfermidades através de suas fezes ressecadas, via respiratória e através de parasitas conhecido como piolho (Labanhare \& Perrelly, 2007; Nunes \& Miranda, 2010). Silva \& Capuano (2008), em um estudo que tinha como objetivo analisar a presença de Cryptococcus e parasitas em fezes dos pombos foi registrado a presença do fungo em $75 \%$ e de parasitas em $32 \%$ e de ambos em $25 \%$, em uma amostra composta por 68 "pools" de excretas presentes em áreas públicas de Ribeirão Preto - SP.

A relação existente entre os pombos urbanos (Columba livia), o fungo Cryptococcus neoformans e a Criptococose vem provocando uma reflexão não apenas científica como também social, até pelo fato dos casos de criptococose estarem elevandose e os dados obtidos serem provenientes de estudos acadêmicos e a criptococose não ser uma doença de notificação compulsória. (Ribeiro, 2019).

Os pombos sendo considerados como domésticos por dividirem de nosso habitat, depositam suas fezes e possuindo o $\mathrm{pH}$ ideal para o crescimento de fungos como o Aspergillus fumigatus, Histoplasma capsulatum, Candida,Trichosporon, Rhodotorula, Hansenula e Cryptococcus são potenciais patogênicos para a saúde humana. (Menezes et al., 2014). Conforme os pesquisadores Marques et al. (2010) os fungos do gênero Fusarium spp infectam plantas e animais, até o homem, sendo responsável por infecções oportunistas que causam lesão na pele, unhas, olhos ou intraperitoneal (peritonite). Segundo Marcondes (2001) os pombos albergam parasitos que podem transmitir doenças ao homem.

Nunes (2003) menciona certas doenças que por sua vez pode ser disseminadas por pombos, destacando algumas delas como: A criptococose; Salmonelose; Encefalites virais; Coliformes Termotolerantes; Toxoplasmose.

A criptococose considerada uma infecção oportunista causada por fungos do gênero Cryptococcus neoformans / C. gattii, transmitida pelos pombos urbanos, encontradas nas fezes, através da inalação dos esporos ele entra aos pulmões afetando o organismo. Este tipo de infecção pode faltar ar, fraqueza e dor pelo corpo. Podendo afetar mais rápido em pacientes soropositivos (Maranhão, et al., 2020). De acordo com Pappalardo \& Melhem (2003) nos humanos, a criptococose considerada mais frequente nos adultos, podendo também afetar as crianças.

Nascimento et al., (2020) cita que a criptococose não é considerada uma doença com maior quantidades de casos em animais e no humano, mas ressaltam a importância do conhecimento epidemiológico relacionados a disseminação com o intuito de prevenção a infecções. E desta forma impede acontecimentos de quadros graves até a morte.

A transmissão da salmonelose ocorre de forma diretamente e indiretamente estando associados com condições de saúde, objetos que estejam contaminados, praticas de manejo bem como a água que possui o agente etiológico, essa doença é considerada responsável por diversos casos de doenças gastrointestinais em humanos (Gomes et al., 2020). Os pesquisadores Hoelzer et al., (2011), relataram que a transmissão da salmonelose pelos pombos para os humanos devem ser mais estudadas, por apresentar em diversas amostras nas fezes dos pombos o agente etiológico.

Os autores Queiroz et al. 2008; Pereira et al. 2008 relataram que as Encefalites virais, podem ser transmitidas por mosquitos que picam os pombos que podem depois picar os seres humanos e passar o vírus acometendo o sistema nervoso. 
Nunes (2003) cita os coliformes Termotolerantes, destacando entre eles a Escherichia Coli, que é considerada um tipo de bactéria que provoca infecção, presente nas excretas dos pombos e no intestino dos humanos. O pesquisador esclarece a importância na higiene, para evitar o possível contagio lavando bem as mãos, pois uma vez o individuo infectado pode ter sintomas como diarreia, dor na região da barriga e vômitos.

A toxoplasmose é considerada uma doença infecciosa através das fezes dos gatos, pelo protozoário Toxoplasma gondii, o parasita desta doença pode afetar principalmente pessoas que estejam com o sistema imunológico enfraquecido, podendo produzir abortos e até mesmo um tipo de resfriado (Gomes \& Sousa, 2020; Rey, 2008).

Em um estudo guiado por Reolon et al., (2004), no qual coletou 88 amostras (excretas de pombos) em pontos distintos da cidade de Porto Alegre - RS, a presença do fungo Cryptococcus neoformans foi identificado em todas as amostras analisadas, comprovando a presença do fungo nas aves que vivem na cidade. Nesta mesma perspectiva Queiroz et al. (2008), em uma amostra constituída por 79 fezes de pombos da cidade de São Paulo - SP, identificaram a presença de C. neoformans em 13, 9 \% da amostra analisada. Em outra pesquisa conduzida na região Centro-oeste brasileiro, Filiú et al., (2002), coletaram 20 amostras de excretas de pombos estando nos distintos ambientes, segundo os autores existiu a ocorrência de C. neoformans em metade das amostras coletadas na cidade de Campo Grande - MS.

Grandes concentrações de pombos em determinada área trazem complicações não só como problema estético e sujidade no local, mas como um risco potencial à saúde pública. As excretas de pombos são consideradas agentes disseminadores de agentes patogênicos, e principalmente Cryptococcus neoformans acometendo os seres humanos. (Reolon et al., 2004). É evidenciada uma crescente no número de pacientes com síndrome da imunossupressão adquirida (SIDA), ao mesmo passo que é constatado maior incidência da criptococose, assim, a enfermidade é representada como oportunista com maior morbidade e mortalidade em portadores de SIDA (Queiroz et al., 2008). Desta forma a via de transmissão mais comum destas zoonoses são as fezes ressecadas em suspensão, que podem ser respiradas. (Ribeiro, 2017; Aguiar et al., 2011).

Teixeira (2003) relata no seu estudo que a educação tem o potencial de instrumentalizar os sujeitos sociais para o enfrentamento as realidades. À construção de vínculos existentes entre a ação médica e o cotidiano social, por isso a conscientização em saúde deve atuar na prevenção e na promoção de conflitos que convergem sumariamente em uma oportunidade de reflexão autônoma e crítica, encorajando os sujeitos sociais a adotarem padrões que visem em nível individual e coletivo o aprimoramento das condições de saúde e de meio ambiente (Vasconcelos, 2001; Junior et al., 2004; Oliveira \& Gonçalves, 2004). Diante de tais estratégias, ainda a educação ambiental e zoonótica e a conscientização da população são as estratégias mais eficientes (Martins, 2015).

Assim, a aglomeração de pombos marcante nas cidades no centro representa um expressivo obstáculo de saúde pública. Ainda que o presente estudo não tenha constatado presença de agentes patogênicos nas fezes de pombos do centro na cidade de Campina Grande - PB, contudo, podem ter sido influenciados pela temperatura. As amostras investigadas foram limitadas, logo, outras pesquisas envolvendo um maior número de coletas devem ser realizadas, no intuito de fornecer dados confiáveis.

\section{Considerações Finais}

Diante da literatura podemos verificar que os pombos urbanos podem disseminar varias doenças para os seres humanos, a grande movimentação dos pombos em locais de acesso ao mesmo habitat dos humanos como no caso nas praças e nos centros urbanos pode ser mencionada como um problema grave na saúde, onde esses pombos podem transmitir as doenças através de suas fezes com a inalação dos esporos presentes na poeira das excretas. Desta forma o contato com estas aves deve ser evitado.

Dentre as diversas doenças de acordo com a literatura científica, destaca-se a Criptococose que é causada pelo fungo Cryptococus neoformans, sendo disseminada através da poeira pela inalação do fungo que estão presentes nas excretas dos 
pombos. Juntamente com a Salmonelose que pode ser adquirida através de alimentos que estejam contaminados pela bactéria e fezes dos pombos.

Deste modo, estudos desenvolvidos tendo por proposito investigar a participação dessas aves na disseminação de enfermidades para o ser humano tem certa importância, no intuito de promover mudanças de hábitos populacionais que por falta de conhecimento acabam por contribuir para uma intensa quantidade reprodutiva de aves marcante nas cidades brasileiras. Apesar desta pesquisa não sustentar a hipótese de que as excretas de pombos da cidade de Campina Grande-PB são polo de agentes patogênicos, cabe destacar que a amostra analisada foi extremamente limitada, de forma que são necessários novos estudos envolvendo maior número de amostras analisadas.

Diante do evidenciado torna-se explícito a realização de programas direcionados para o controle do manejo de aves que apresentam intensa agitação de pessoas, tendo em vista da urgência de medidas voltadas para prevenir os perigos que estas aves podem causar para a saúde humana, cabe salientar que os humanos são agentes de grande importância no processo de reprodução destas aves, visto que muitas das vezes fornecem alimentos para os animais, assim, a conscientização da população pode ser encarada como uma das formas de reduzir a manifestação de pombos nos centros urbanos.

Como proposta de trabalhos futuros, sugerimos a replicação do nosso trabalho com uma quantidade maior de amostra. Também sugerimos a investigação em diferentes regiões e uma análise investigativa de associação dos agentes patogênicos em indivíduos.

\section{Agradecimentos}

Os autores agradecem a Universidade Estadual da Paraíba - UEPB ao Grupo de Pesquisa em Tratamentos Avançados de Águas - GRUTAA/UEPB, ao CNPq e CAPES pelas bolsas concedidas.

\section{Referências}

Aguiar, M. B., Luciano, L., \& Beijamini, V. (2011). Avaliação dos riscos de contaminação relacionados com a superpopulação de Columbia livia (pombos) em trabalhadores portuários avulsos. Revista Brasileira de Pesquisa em Saúde/Brazilian Journal of Health Research.

Aguiar, P. A. D. F. D., Pedroso, R. D. S., Borges, A. S., Moreira, T. D. A., Araújo, L. B. D., \& Röder, D. V. D. D. B. (2017). The epidemiology of cryptococcosis and the characterization of Cryptococcus neoformans isolated in a Brazilian University Hospital. Revista do Instituto de Medicina Tropical de São Paulo, 59.

Araújo, D. D., da Silva, R. E., da Silva Pereira, H., Costa, D. S., de Sá, R. E., da Silva Neto, A. R., ... \& Fonseca, F. M. (2021). Avaliação da diversidade genética de amostras de Cryptococcus neoformans isoladas em diferentes regiões geográficas. Research, Society and Development, $10(9)$, e51410918333-e51410918333.

Albano, A. P. N. (2009). Fungos e micoses em animais silvestres recebidos por Centros de Triagem (Master's thesis, Universidade Federal de Pelotas).

Amâncio, S., SouzA, V. D., \& Melo, C. (2008). Columba livia e Pitangus sulphuratus como indicadoras de qualidade ambiental em área urbana. Revista Brasileira de Ornitologia, 16(1), 32-37

Araújo, C. D., de Carvalho, F. G., \& De Albuquerque, L. B. (2000). Levantamento epidemiológico das Zoonoses transmitidas por pombos em Campo GrandeMS. Multitemas.

Beck, P. V. (2003). Estudo das infestações de pombos nas edificações da cidade de Brasília.

Barbosa Junior, A. M., Santos, B. F. D. O., Carvalho, E. D. O., Mélo, D. L. F. M. D., Trindade, R. D. C., \& Stoianoff, M. A. D. R. (2013). Biological activity of Cryptococcus neoformans and Cryptococcus gattii from clinical and environmental isolates. Jornal Brasileiro de Patologia e Medicina Laboratorial, 49, 160168.

Contin, J. T., Quaresma, G. D. S., Silva, E. F. D., \& Linardi, V. R. (2011). Ocorrência de Cryptococcus neoformans em fezes de pombos na cidade de Caratinga, MG-Brasil. Revista Médica de Minas Gerais, 21(1), 19-24.

Cruz, G. De M., Junior, De N. N. C., \& Silva, J. X. (2015). Incidência de cryptococcus neoformans em aves de cativeiro e aves de vida livre. Revista de Divulgação Científica Sena Aires, 3(2), 161-168.

Carrasco, A. D. O. T., Issakowicz, J. C., de Morais, M. T. G. F., Fatoretto, L. A., Pandolfi, J. R. C., da Silva, L. C., \& Pinto, A. A. (2011). Levantamento Sorológico de Mycoplasma spp, Salmonella sp e Doença de Newcastle em Pombos Domésticos (Columba livia) de Vida Livre. Journal of Health Sciences, 13(1).

Farias, J. N. B. (2016). Percepção popular sobre os pombos (Columba livia) como animais sinantrópicos no agreste paraibano, Nordeste do Brasil. 
Filiú, W. F. D. O., Wanke, B., Agüena, S. M., Vilela, V. O., Macedo, R. C. L., \& Lazéra, M. (2002). Cativeiro de aves como fonte de Cryptococcus neoformans na cidade de Campo Grande, Mato Grosso do Sul, Brasil. Revista da Sociedade Brasileira de Medicina Tropical, 35, 591-595.

Fraga, C. F. (2014). Ocorrência de doenças micóticas em aves silvestres no Brasil: revisão bibliográfica.

Gomes, V. Da S., De Souza, D. P. M., \& De Sousa, N. P. R. (2020). Pombos urbanos columba livia como agentes transmissores de infecções na cidade de araguaína-to. Facit Business and Technology Journal, 1(20).

Hoelzer, K., Switt, A. I. M., \& Wiedmann, M. (2011). Animal contact as a source of human non-typhoidal salmonellosis. Veterinary research, 42(1), 1-28.

Junior, H. De. A., Souza, M. A. D., \& Brochier, J. I. (2004). Representação social da educação ambiental e da educação em saúde em universitários. Psicologia: Reflexão e Crítica, 17, 43-50.

Kahn, R. E., Morozov, I., Feldmann, H., \& Richt, J. A. (2012). 6th international conference on emerging zoonoses. Zoonoses and public health, $59,2-31$.

Labanhare, L. L., \& de Souza Perrelli, M. A. (2007). Pombos urbanos: biologia, ecologia e métodos de controle populacional. Multitemas.

Leite, D. P., Amadio, J. V., Martins, E. R., Simões, S. A., Yamamoto, A. C. A., Leal-Santos, F. A., ... \& Hahn, R. C. (2012). Cryptococcus spp isolated from dust microhabitat in Brazilian libraries. Journal of Occupational Medicine and Toxicology, 7(1), 1-7.

Martins, C. M., Biondo, A. W., Braga, K. F., \& Oliveira, S. T. (2016). Percepção de usuários de espaços públicos de Curitiba, Paraná, sobre a presença de pombos (Columba livia). Archives of Veterinary Science, 20(4).

Mezzari, A., Wiebbelling, A. M. P., Wenczenovicz, C., Souza, C. D. A. D., Freitas, G. S. D. O., Barboza, L. D., ... \& Behar, P. R. P. (2014). Presença do Cryptococcus spp. nas excretas de pombos nos arredores de Hospitais de Porto Alegre.

Moutinho, F. F. B., Serra, C. M. B., Valente, L. C. M., Borges, F. V. B., \& de Faria Neto, F. (2015). Distribuição espaço-temporal das reclamações sobre pombos (columba livia domestica) efetuadas ao centro de controle de zoonoses de niterói, RJ (2009-2013). Hygeia-Revista Brasileira de Geografia Médica e da Saúde, 11(21), 49-61.

Moutinho, F. F. B., Rocha, M. R. D., Borges, F. V. B., Pereira, A. G., \& Serra, C. M. B. (2013). Reclamaçães da comunidade à Seção de Controle de População Animal do Centro de Controle de Zoonoses de Niterói, Rj, Brasil, no período 2006-2010. Revista Brasileira de Ciência Veterinária, 20(1).

Menezes, T., Scain, G., de Quadros, R. M., Miletti, L. C., Souza, A. L., de Lima Miguel, R., \& Marques, S. M. T. (2014). Cryptococcus spp. em excretas de pombos (Columba livia) de áreas públicas de Lages, Santa Catarina. Science and Animal health, 2(2), 102-114.

Miranda, C., Ladendorff, N., \& Knöbl, T. (2014). Percepção da população sobre a participação dos pombos (Columba livia domestica) na transmissão de zoonoses. Atas de Saúde Ambiental-ASA (ISSN 2357-7614), 2(1), 23-28.

Maranhão, F. C. A., Fonseca, D. L., Santos, G. S., Fonseca, L. A. V., Santos, E. J. L., \& Silva, D. M. W. (2020). Disseminated and cutaneous cryptococcosis by C. neoformans VNI in an immunocompetent patient. Jornal Brasileiro de Patologia e Medicina Laboratorial, 56.

Marques, T., Silveira, L. A. M., D'Amorim, M. F. G., Pelli, A., Moura, R. S., Marson, J. M., SILVEIRA, L. A. M. da; D’AMORIM, M. F. G. \& Terra, A. P. S. (2010). Avaliação da microbiota associada à Pseudolynchia canariensis coletadas em pombos domésticos (Columba livia). Revista de Ciências Médicas e Biológicas, 9(3), 224-228.

Martins, R Andrade de. (2012). A origem dos pombos domésticos na estratégia argumentativa de Charles Darwin. Filosofia e História da Biologia, 7(1), 91116.

Martins, C. M., Biondo, A. W., Braga, K. F., \& Oliveira, S. T. (2016). Percepção de usuários de espaços públicos de Curitiba, Paraná, sobre a presença de pombos (Columba livia). Archives of Veterinary Science, 20(4).

Marcondes, C. B. (2001). Flebotomíneos. Entomologia Médica e Veterinária. Editora Atheneu, São Paulo, Rio de Janeiro, Belo Horizonte.

Nascimento, M. A., Santos, E. C. M., de Carvalho, V. M., Borges, M. S., Ederli, J. P. B., de Albuquerque, C. A. N., \& Moris, D. V. (2020). Perfil de pacientes com criptococose em hospital regional do interior de São Paulo. Research, Society and Development, 9(9), e598997642-e598997642.

Neto, J. B. de L., Lopes, D. L., de Souza, F. A. P., dos Santos Pereira, I., \& Vandesmet, L. C. S. (2020). incidência de fungos patogênicos nas fezes de pombos em áreas públicas de quixadá, ceará. Mostra Científica em Biomedicina, 4(2).

Nunes, V. D. F. P. (2003). Pombos urbanos: o desafio de controle. Biológico, 65(1/2), 89-92.

Nunes, J. R. D. S., \& Miranda, D. B. (2010). Aspectos Biológicos De Columba Livia Doméstica (Columbiformes, Columbidae) No Campus Da Universidade De Cuiabá-Unic, Cuiabá-Mt. UNICiências, 14(1).

Oliveira, A. E. D. S., \& Machado, C. J. S. (2009). Quem é quem diante da presença de espécies exóticas no Brasil? Uma leitura do arcabouço institucional-legal voltada para a formulação de uma Política Pública Nacional. Ambiente \& Sociedade, 12, 373-387.

Pizani, A. T., \& dos Santos, M. O. (2017). Criptococose em pacientes HIV positivos: revisão sistemática da literatura. Revista Saúde UniToledo, 1(1).

Pappalardo, M. C., \& Melhem, M. S. (2003). Cryptococcosis: a review of the Brazilian experience for the disease. Revista do Instituto de Medicina Tropical de São Paulo, 45, 299-305.

Pereira, P. Pereira P; Sabadin JC; Wolfran L; Osaki SC. Medvep Dermato-Revista de Educação Continuada em Dermatologia e Alergologia Veterinária; 5 (14); 6-9.

Queiroz, João Paulo Araújo Fernandes De; Sousa, Francisco David Nascimento; Lage, Regiana Araújo; Izael, Marisa De Alencar; Santos, Anderson Gurgel Dos. (2008). Criptococose-uma revisão bibliográfica. Acta Veterinaria Brasilica, 2(2), 32-38. 
Research, Society and Development, v. 10, n. 10, e387101018717, 2021

(CC BY 4.0) | ISSN 2525-3409 | DOI: http://dx.doi.org/10.33448/rsd-v10i10.18717

Ribeiro, C. L. (2019). Criptococose e pombos urbanos (Columba livia): uma reflexão social, ambiental e de políticas públicas. Multitemas, 205-222.

Ribeiro, M. F. D. P., Silva, A. D. M. D., Fernandes, W. S., \& Mello, M. M. D. (2017). Isolamento de Cryptococcus neoformans em fezes de pombos (Columba livia) em praças públicas de São José dos Campos-SP. J. Health Sci. Inst, 23-27.

Rey, L. (2008). Parasitologia: parasitos e doenças parasitárias do homem nos trópicos ocidentais. In Parasitologia: parasitos e doenças parasitárias do homem nos Trópicos Ocidentais (pp. 883-883).

Reolon, A., Perez, L. R. R., \& Mezzari, A. (2004). Prevalência de Cryptococcus neoformans nos pombos urbanos da cidade de Porto Alegre, Rio Grande do Sul. Jornal Brasileiro de Patologia e Medicina Laboratorial, 40, 293-298.

Silva, S. P., Costa, C. B. L., Silva, J. D. F., Alves, R. R. V., Silva, G. A. S., Freitas, A. F. S, \& Napoleão, T. H. (2021). Mecanismos de resistência de Cryptococcus spp. e compostos de plantas como ferramentas para combatê-los. Research, Society and Development, 10 (2), e57810212819-e57810212819.

Schuller, M. (2005). Pombos urbanos: um caso de saúde pública. Sociedade Brasileira de Controle de Contaminação, 29, 32-37.

Silva, J. O., \& Capuano, D. M. (2008). Ocorrência de Cryptococcus spp e de parasitas de interesse em saúde pública, nos excretas de pombos na cidade de Ribeirão Preto, São Paulo, Brasil. Revista do Instituto Adolfo Lutz (Impresso), 67(2), 137-141.

Torres, A. C. D., D'Aparecida, N. S., \& Haas, D. J. (2015). Principais zoonoses víricas, fúngicas e parasitárias de aves domésticas e silvestres. revista veterinária em foco, 13(1).

Teixeira, P. M. M. (2003). A educação científica sob a perspectiva da pedagogia histórico-crítica e do movimento CTS no ensino de ciências. Ciência \& Educação (Bauru), 9(2), 177-190.

Takahara, D. T. (2011). Isolamento e identificação de Cryptococcus neoformans a partir de excretas de pombos provenientes de locais públicos e residênciais de Cuiabá e Várzea Grande-MT. 122 pth edition. Cuiabá/MT:(MSc. Dissertation. Faculdade de Ciências Médicas-Universidade Federal de Mato Grosso.

Vasconcelos, E. M. (2001). Redefinindo as práticas de saúde a partir de experiências de educação popular nos serviços de saúde. Interface-Comunicação, Saúde, Educação, 5, 121-126. 